\title{
A Multisine Approach for Trajectory Optimization Based on Information Gain
}

\author{
L. Mihaylova, J. De Schutter and H. Bruyninckx \\ Katholieke Universiteit Leuven, Department of Mechanical Engineering \\ Division of Production Engineering, Machine Design and Automation, \\ Celestijnenlaan 300B, B-3001 Heverlee Belgium \\ E-mail:Lyudmila.Mihaylova@mech.kuleuven.ac.be, Fax: +32 16322987
}

\begin{abstract}
This paper presents a multisine approach for trajectory optimization based on information gain, with distance and orientation sensing to known beacons. It addresses the problem of active sensing, i.e. the selection of a robot motion or sequence of motions, which make the robot arrive in its desired goal configuration (position and orientation) with maximum accuracy, given the available sensor information. The optimal trajectory is parameterized as a linear combination of sinusoidal functions. An appropriate optimality criterion is selected which takes into account various requirements (e.g. maximum accuracy and minimum time). Several constraints can be formulated, e.g. with respect to collision avoidance. The optimal trajectory is then determined by numerical optimization techniques. The approach is applicable to both nonholonomic and holonomic robots. Its effectiveness is illustrated here for a nonholonomic wheeled mobile robot (WMR) in an environment with and without obstacles.
\end{abstract}

Keywords: active sensing, mobile robots, uncertainty, trajectory generation, information gain

\section{Motivation}

Many applications in mobile robotics have sophisticated motion requirements. A successful task completion is often related to reaching quite accurately a goal while processing noisy sensor data under uncertainties. This refers to active sensing, the main question of which is: "What motion should the robot execute in order to gain (the most accurate) information about its environment?". Active sensing is a challenging topic for different reasons. The solution depends on the optimality criterion. This should be such that maximum information is extracted from the sensor data. At the same time computationally efficient sensor data processing is needed for the on-line execution of the generated motions. Obstacle avoidance adds an another level of difficulty [12]. Uncertainty (e.g. in the model and sensor data) call for stochastic estimation and control techniques. The nonlinear character of the problem adds further difficulty to this. Finally, for nonholonomic systems nonholonomic constraints have to be taken into account, so that the generated path from the admissible configuration space corresponds to a feasible trajectory [22], [13].

This paper introduces an approach for trajectory optimization based on information gain. A mobile robot is moving in the Cartesian plane starting from an initial configuration to a desired goal configuration which has to be reached with maximum accuracy. While moving around, the robot can make use of uncertain sensor data to improve the estimate of its configuration (position and orientation). The sensor data consist of distance and orientation measured with respect to one or more beacons. Hence, the optimization problem consists in finding the path that minimizes the uncertainty of the configuration estimate at the goal configuration.

Between two points there are an infinite number of trajectories. The key idea is to represent them by a parameterized family of possible trajectories. This way the infinite-dimensional optimization problem is reduced to a finite-dimensional, parameterized optimization problem. We propose to use multisine trajectories: deviations from a nominal trajectory are represented by linear combinations of a limited number of sines. Active sensing is considered as a global optimization problem subject to constraints. 
Several optimization criteria are possible, such as entropy [23] or a weighted trace of the covariance matrix, respectively the Fisher information matrix at the final configuration estimate. The covariance matrix results from a stochastic estimator: standard linear Kalman filter (KF) [11], extended KF [1], unscented KF [10], or other Kalman filtering technique.

One feature of our approach is that the optimal trajectory is generated as a linear combination of sine functions. This parameterization is appealing because: 1) the complete path can be characterized by a limited number of parameters; 2) all primitives satisfy, at least partially, the boundary conditions in initial and goal configuration; 3) the harmonic content of the motion, hence the maximum maneuvering frequency, is explicitly kept under control. On the other hand, the disadvantage - as opposed to e.g. splines - is that it is a global parameterization. In particular, modifications of the path, e.g. to avoid an obstacle, have a global effect. Multisines are rich excitation signals which are often used in experimental system identification $[24,6]$. And in fact, system identification and active sensing can be considered as one and the same problem. Multisines have also proved to offer advantages for nonholonomic control systems (they assure smooth stabilization), which could be interesting if tracking control is added later on to the estimation problem considered here. It should be noted that within our approach other parameterizations are also possible.

\subsection{Related Work}

Active sensing corresponds to a constrained optimization of a multi-objective criterion with respect to a sequence of actions. Different kinds of problems arise and the methods for their solution can be classified in two groups: $i)$ the sequence of actions is restricted to a parametrized trajectory or trajectory represented by some functions [4], [12], [18], [2], [3]. To this group belongs the approach, proposed in the present paper. In [7] the choice of "where to look next" is treated as a special case of an optimal experiment design. Minimization of the weighted trace of the estimation error covariance matrix is chosen as a criterion to perform the next motion. ii) The sequence of actions is not reduced to a parametrized trajectory. Then Markov decision processes (MDPs) and partially observable MDPs optimization problems need to be solved $[25,5]$. The robot actions and the environment are modeled in a statistical framework. Solutions proposed in $[21,5,8]$ are based on minimization of a probabilistic metric (entropy). The method developed in [5] is applied under positional uncertainty; the approach proposed here is suitable under both positional and orientation uncertainty.

The rest of the paper is structured as follows. Section 2 describes the motion and measurement models of the considered nonholonomic WMR. The proposed approach for trajectory optimization is introduced in Section 3. Simulation studies illustrating its effectiveness are presented in Section 4. Finally Section 5 discusses the results and Section 6 summarizes the conclusions.

\section{Motion and Measurement Models}

The following active sensing task is considered. The WMR starting from a configuration (position and orientation) $\left(x_{s}, y_{s}, \phi_{s}\right)^{T}$ is required to reach a desired goal configuration $\left(x_{g}, y_{g}, \phi_{g}\right)^{T}$ moving around a known, nominal reference trajectory $\left(x_{r, k}, y_{r, k}, \phi_{r, k}\right)^{T} . k$ represents the discrete time index. The actually executed, modified trajectory is determined by $\left(x_{k}, y_{k}, \phi_{k}\right)^{T}$. The WMR generates its actions by processing measurement data originating from one or more beacons.

The vehicle motion is described by the model [17]

$$
\left(\begin{array}{c}
x_{k+1} \\
y_{k+1} \\
\phi_{k+1}
\end{array}\right)=\left(\begin{array}{c}
x_{k}+v_{k} \Delta T \cos \left(\phi_{k}+\psi_{k}\right) \\
y_{k}+v_{k} \Delta T \sin \left(\phi_{k}+\psi_{k}\right) \\
\phi_{k}+\frac{v_{k} \Delta T}{L} \sin \psi_{k}
\end{array}\right)+\left(\begin{array}{c}
\eta_{x, k} \\
\eta_{y, k} \\
\eta_{\phi, k}
\end{array}\right),
$$

where $x_{k}$ and $y_{k}$ denote the WMR position coordinates with respect to a fixed frame (Fig. 1), and $\phi_{k}$ represents the orientation angle with respect to the $x$ axis. They form the state vector $\boldsymbol{x}_{k}=\left(x_{k}, y_{k}, \phi_{k}\right)^{T} . L$ is the wheel base (the distance between the front steering wheel and the axis of the driving wheels), $\Delta T$ is the sampling time, and $\boldsymbol{\eta}_{k}=\left(\eta_{x, k}, \eta_{x, k}, \eta_{\phi, k}\right)^{T}$ represents the process noise. The WMR is controlled through a demanded velocity $v_{k}$ and a direction of travel $\psi_{k}$, i.e. the control vector is $\boldsymbol{u}_{k}=\left(v_{k}, \psi_{k}\right)^{T}$. Due to physical 


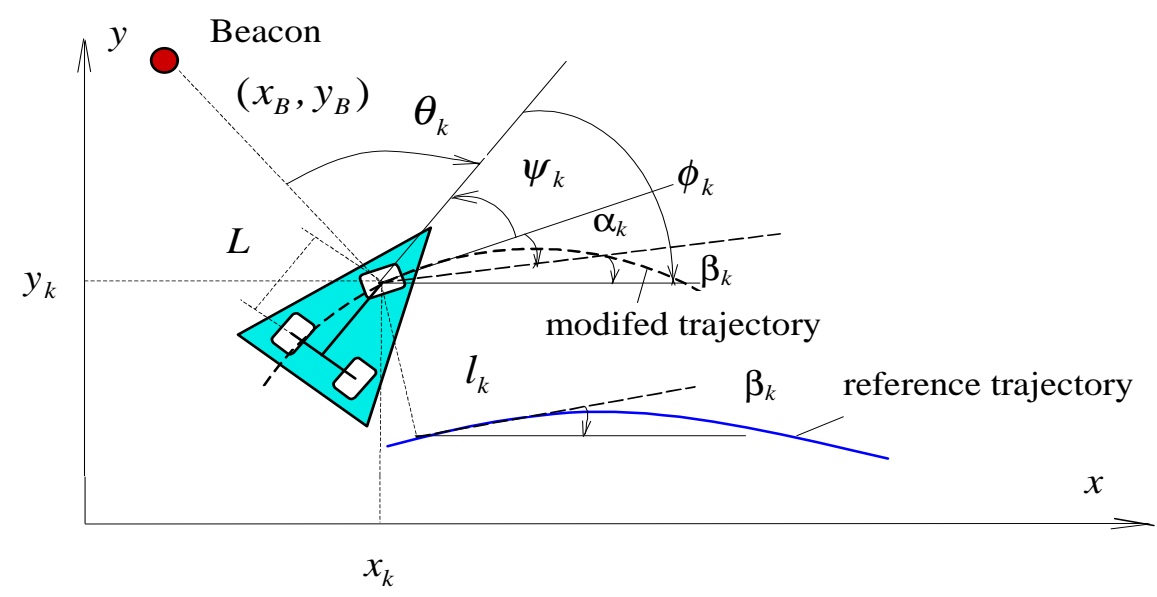

Figure 1: WMR coordinates

constraints, both the velocity $v_{k}$, and the angle $\psi_{k}$ cannot exceed boundary values, namely $v_{k} \in\left[0, v_{\max }\right]$, $\psi_{k} \in\left[-\psi_{\max }, \psi_{\max }\right]\left(\psi_{\max } \leq \frac{\pi}{2}\right)$. The WMR can only perform forward motions.

The vehicle is equipped with a sensor measuring the range $r_{k}$ and bearing $\theta_{k}$ to a beacon $B$, located at known coordinates, $\left(x_{B}, y_{B}\right)^{T}$. The observation equation for the beacon is given by [17]

$$
\left(\begin{array}{c}
r_{k} \\
\theta_{k}
\end{array}\right)=\left(\begin{array}{c}
\sqrt{\left(x_{B}-x_{k}\right)^{2}+\left(y_{B}-y_{k}\right)^{2}} \\
\arctan \left(\frac{y_{B}-y_{k}}{x_{B}-x_{k}}\right)-\phi_{k}
\end{array}\right)+\left(\begin{array}{c}
\xi_{r, k} \\
\xi_{\theta, k}
\end{array}\right)
$$

where $\boldsymbol{\xi}_{k}=\left(\xi_{r, k}, \xi_{\theta, k}\right)^{T}$ is the observation noise. The measurement vector is further denoted by $\boldsymbol{z}_{k}=$ $\left(r_{k}, \theta_{k}\right)^{T}$. The noise vectors $\boldsymbol{\eta}_{k}$ and $\boldsymbol{\xi}_{k}$ are assumed Gaussian, zero mean, mutually uncorrelated, with covariances $\boldsymbol{Q}_{k}$ and $\boldsymbol{R}_{k}$, respectively.

The relative location of the beacon with respect to the moving WMR is of paramount importance for the active sensing task, for providing accurate and informative sensor data. If more $\left(n_{B}\right)$ beacons are available, the observation equation acquires the form

$$
\left(\begin{array}{c}
r_{1, k} \\
\theta_{1, k} \\
\vdots \\
r_{n_{B}, k} \\
\theta_{n_{B}, k}
\end{array}\right)=\left(\begin{array}{c}
\sqrt{\left(\tilde{x}_{1, k}\right)^{2}+\left(\tilde{y}_{1, k}\right)^{2}} \\
\arctan \left(\frac{\tilde{y}_{1, k}}{\tilde{x}_{1, k}}\right)-\phi_{k} \\
\vdots \\
\sqrt{\left(\tilde{x}_{n_{B}, k}\right)^{2}+\left(\tilde{y}_{n_{B}, k}\right)^{2}} \\
\arctan \left(\frac{\tilde{y}_{n_{B}, k}}{\tilde{x}_{n_{B}, k}}\right)-\phi_{k}
\end{array}\right)+\left(\begin{array}{c}
\xi_{r_{1}, k} \\
\xi_{\theta_{1}, k} \\
\vdots \\
\xi_{n_{n_{B}}, k} \\
\xi_{\theta_{n_{B}}, k}
\end{array}\right),
$$

where $\tilde{x}_{i, k}=x_{B_{i}}-x_{k}, \quad \tilde{y}_{i, k}=y_{B_{i}}-y_{k}, \quad i=1, \ldots, n_{B}$ and with a measurement covariance matrix

$$
R_{k}=\operatorname{diag}\left\{R_{1, k}, \ldots, R_{n_{B}, k}\right\} .
$$

\section{Trajectory Optimization}

Consider the case when the optimization starts from a known, nominal reference trajectory between the starting and the goal configuration.

The problem of determining the 'best' trajectory $\boldsymbol{q}^{*}$ with respect to an optimality criterion $J$ can be formulated as to find

$$
\boldsymbol{q}^{*}=\operatorname{argmin}(J)
$$

subject to constraints

$$
\left|c_{i, k}\right| \leq c_{i, t h r}
$$




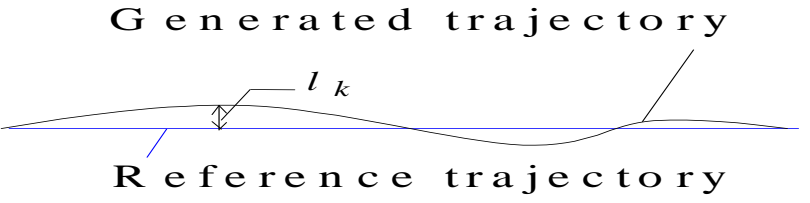

Figure 2: Lateral Deviation $l_{k}$

where the variables $c_{i, k}, i=1, \ldots, r$ are bounded by threshold values $c_{i, t h r}$ (e.g. maximal lateral deviation from a reference trajectory, maximal velocity and acceleration, maximal steering angle, minimum distance to obstacles, etc.). The optimality criterion to be minimized is a measure of the accuracy of the robot's configuration estimate.

The approach is based on a parametrization of the class

$$
\mathcal{Q}=\mathcal{Q}(\boldsymbol{p}), \quad \boldsymbol{p} \in \mathcal{P},
$$

of harmonic functions, where $\boldsymbol{p}$ is a vector of parameters obeying to preset physical constraints. With $N$ the number of harmonic functions, the new (modified) robot trajectory is generated on the basis of the reference one by the lateral deviation $l_{k}$ (lateral is the orthogonal robot motion deviation from the reference trajectory in $y$ direction, Fig. 2) as a linear superposition

$$
l_{k}=\sum_{i=1}^{N} A_{i} \sin \left(i \pi \frac{s_{r, k}}{s_{r, t o t a l}}\right)
$$

of sinusoids, the amplitudes $A_{i}$ of which are constants, $s_{r, k}$ is the path length up to instant $k, s_{r, t o t a l}$ is the total path length, and $r$ refers to the reference trajectory. Expressed as a function of the time, the lateral deviation is

$$
l_{k}=\sum_{i=1}^{N} A_{i} \sin \left(i \pi \frac{t_{k}}{t_{\text {total }}}\right),
$$

where $t_{k}$ is the current time, and $t_{\text {total }}$ is a preset time for reaching the goal configuration.

Clearly, the problem described above can be cast into the problem of trajectory generation of a system described by equations of the form

$$
\begin{aligned}
& \boldsymbol{x}_{k+1}=f\left(\boldsymbol{x}_{k}, \boldsymbol{u}_{k}, \boldsymbol{\eta}_{k}\right) \\
& \boldsymbol{z}_{k+1}=h\left(\boldsymbol{x}_{k+1}, \boldsymbol{\xi}_{k+1}\right)
\end{aligned}
$$

with $f$ and $h$ nonlinear functions. In this formulation active sensing is a global optimization problem (on the whole robot trajectory) with a criterion to be minimized

$$
J=\min _{A_{i, k}}\left\{a_{1} \mathcal{U}+a_{2} \mathcal{C}\right\}
$$

under constraints (2). $\mathcal{U}$ characterizes the expected uncertainty to be minimized (or information extraction and accuracy to be maximized), $\mathcal{C}$ is the cost part. As $\mathcal{U}$ could be chosen any information function, e.g. the entropy, or a scalar function of the covariance matrix of the estimated states. Information functions (like the logarithm of the determinant of the covariance matrix and the maximum eigenvalue of the covariance matrix) possess the properties of nonnegativity, concavity, etc. that make them suitable for ranking [7].

Here $\mathcal{U}$ is in the form

$$
\mathcal{U}=\operatorname{trace}(\boldsymbol{W} \boldsymbol{P}),
$$

where $\boldsymbol{P}$ is the covariance matrix of the estimated states (at the goal configuration), $\boldsymbol{W}$ is a weighting matrix; $\operatorname{trace}(\cdot)$ denotes the matrix trace; $a_{1}$ and $a_{2}$ above are dimensionless positive weighting constants, 
giving different weight to the information and cost terms of $J . \mathcal{C}$ accounts for losses such as time, traveled distance, etc. Here $\mathcal{C}$ is assumed to be the relative time

$$
\mathcal{C}=t_{\text {total }} / t_{r, \text { total }},
$$

where $t_{\text {total }}$ is the total time for reaching the goal configuration on the modified trajectory versus the respective time $t_{r, \text { total }}$ over the reference trajectory. Minimization of $J$ with respect to parameters of the modified trajectories guarantees trajectories with minimal uncertainty bounds. Within a statistical framework the covariance matrix $\boldsymbol{P}$ represents an information criterion. The weighting matrix $\boldsymbol{W}$ is presented as a product of a normalizing matrix $\boldsymbol{N}$, and a scaling matrix $\boldsymbol{M}, \boldsymbol{W}=\boldsymbol{M} \boldsymbol{N}$. The normalizing matrix $\boldsymbol{N}=\operatorname{diag}\left\{1 / \sigma_{1}^{2}, 1 / \sigma_{2}^{2}, \ldots, \sigma_{n}^{2}\right\},(n$ is the state vector dimension) transforms the criterion into a measure which is invariant to different physical units. $\sigma_{i}, i=1, \ldots, n$, are assumed here to be the standard deviations at the goal configuration on the reference trajectory. Depending on the particular task, they could be chosen otherwise. The scaling matrix $\boldsymbol{M}=\operatorname{diag}\left\{m_{1}, \ldots, m_{n}\right\}$ gives different weights to the separate terms of the trace. Without loss of generality, it is assumed that $\sum_{i=1}^{n} m_{i}=n$. The criterion $J$ is a dimensionless scalar. As 'good' are considered trajectories which at the goal configuration have $\mathcal{U} \in[0, n]$, i.e. less than for the reference trajectory.

When importance is given to accuracy of both the goal configuration, and the intermediate configurations, the optimization could be performed by averaging over an interval $\left[k_{a}, k_{b}\right]$,

$$
\mathcal{U}=\frac{1}{k_{b}-k_{a}} \sum_{k=k_{a}}^{k_{b}} \operatorname{trace}\left(\boldsymbol{W}_{\boldsymbol{k}} \boldsymbol{P}_{k}\right)
$$

The $\sigma_{i}$, participating in the normalizing matrix $\boldsymbol{N}$ could then be the average standard deviations with averaging performed on the whole reference trajectory or over a part of it. Other choices of $\sigma_{i}$ are possible, depending on the task.

For the considered WMR the constraints (2) are of the form

$$
\begin{aligned}
& \left|l_{k}\right| \leq l_{\max }, \\
& v_{k} \leq v_{\max }, \\
& \left|\psi_{k}\right| \leq \psi_{\max }, \\
& \left|\phi_{\text {end }}-\phi_{g}\right| \leq \phi_{\text {error }}, \\
& d_{o_{j}, k} \geq d_{o_{j}, \text { min }},
\end{aligned}
$$

where $l_{k}$ is the lateral deviation of the optimal trajectory from the reference one, $l_{\max }$ is the maximum allowed lateral deviation. A constraint is introduced for the goal orientation angle, $\phi_{g}$. The WMR orientation angle $\phi_{\text {end }}$ at the end of the path should be close to $\phi_{g}$, with accuracy, preset by an acceptable error $\phi_{\text {error }}$. The distance $d_{o_{j}, k}=\sqrt{\left(x_{k}-x_{o, j}\right)^{2}+\left(y_{k}-y_{o, j}\right)^{2}}$ from the WMR to the $j$-th obstacle, $j=1,2, \ldots, n_{o b s}$ $\left(x_{o, j}\right.$ and $y_{o, j}$ are the obstacle coordinates, $n_{o b s}$ is the number of obstacles) should be bigger than a preset threshold $d_{o_{j}, \min }$. Other constraints could also be taken into account in a similar way, e.g. about the rate of change of $v_{k}$, the rate of change of $\psi_{k}$, or some of the constraints could be omitted, $[15,16]$. The problem of trajectory optimization without a reference trajectory can be solved in a similar way to the case with a reference trajectory.

\section{Simulation Studies}

This Section presents simulation results showing the performance of the multisine approach.

The state estimation used throughout the present paper is based on the Unscented Kalman Filter (UKF) [10], [26]. The unscented transformation approximates a probability distribution based on a small number of deterministically chosen test points, referred to as sigma points which have the same first, second and possibly higher moments, as the estimate. The UKF is implemented here in its form with an augmented state vector (a concatenation of the states and the noises) [26]. The sigma points and their weights are 
calculated using the more accurate scaled Unscented Transform [9]. It does not require linearization, nor explicit calculation of Jacobians and Hessians and it is numerically stable due to its factorization-based form.

Example 1. The WMR is moving in the presence of a reference trajectory straight line in an obstaclefree environment between starting and goal configurations, respectively $\boldsymbol{x}_{s}=(1 \mathrm{~m}, 15 \mathrm{~m}, 0 \mathrm{deg})^{T}, \boldsymbol{x}_{g}=$ $(12.84 \mathrm{~m}, 15 \mathrm{~m}, 0 \mathrm{deg})^{T}$ with a constant velocity $v=0.12 \mathrm{~m} / \mathrm{sec}$. The beacon is located in a point with coordinates $x_{B}=9 \mathrm{~m}, y_{B}=19 \mathrm{~m}$. It is assumed that $v_{\max }=0.2 \mathrm{~m} / \mathrm{sec}, \psi_{\max }=60 \mathrm{deg}, l_{\max }=3 \mathrm{~m}$ and $L=0.5 \mathrm{~m}$.

The UKF is implemented with the following parameters, recommendable for systems with Gaussian noises and of order $n=3$ (so that $\kappa+n=3$ ) [9, 26] : $\alpha=1, \beta=2, \kappa=0$. The sigma points and their weights are calculated using the Scaled Unscented Transformation [9], [26]. The initial state estimate vector and covariance matrix are: $\hat{\boldsymbol{x}}_{0 / 0}=(1 \mathrm{~m}, 15 \mathrm{~m}, 0 \mathrm{deg})^{T}$ and $\boldsymbol{P}_{0}=\operatorname{diag}\left\{0.3 \mathrm{~m}^{2}, 0.3 \mathrm{~m}^{2}, 0.0025 \mathrm{deg}^{2}\right\}$. The system and measurement noise covariance matrices are taken as

$$
\begin{aligned}
& \boldsymbol{Q}_{k}=\operatorname{diag}\left\{10^{-6} \mathrm{~m}^{2}, 10^{-6} \mathrm{~m}^{2}, 10^{-4} \mathrm{deg}^{2}\right\}, \\
& \boldsymbol{R}_{k}=\operatorname{diag}\left\{0.0004 d_{k}^{2} \mathrm{~m}^{2}, 100 \mathrm{deg}^{2}\right\},
\end{aligned}
$$

where $d_{k}$ is the distance from the WMR to the beacon. Measured distances $d_{k}$ are used for simulating the measurements, estimated values are used in the UKF (like in [20]). The covariance matrix $\boldsymbol{P}_{k}$ of the estimation error $\boldsymbol{x}_{k}-\hat{\boldsymbol{x}}_{k}$ defines an uncertainty ellipsoid $\left(\boldsymbol{x}_{k}-\hat{\boldsymbol{x}}_{k}\right)^{T} \boldsymbol{P}_{k}\left(\boldsymbol{x}_{k}-\hat{\boldsymbol{x}}_{k}\right)=1$ that, with respect to the positions only represents a confidence ellipse, characterizing the performance of the active sensing.

The reference trajectory and modified trajectory, generated with different numbers of sinusoids $N$ (in accordance with (4), criterion (8) with terms (9) and (10) ) together with the uncertainty ellipses are shown on Fig. 3.

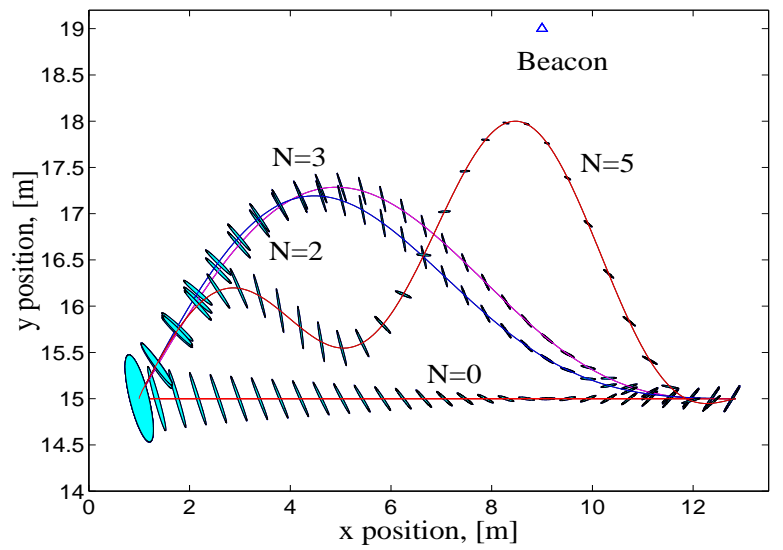

Figure 3: Trajectories, generated with (9) and (10), and different number $N$ of sine terms. Uncertainty ellipses plotted around the trajectories.

The evolution in time of the weighted covariance trace is presented in Fig. 4. The multisine approach gives faster increase of the information at the beginning of the modified trajectories, than those on the straight-line. Constraints (12)-(15) are taken into account. The normalizing and scaling matrices in the criterion $J$ are chosen in the form: $\boldsymbol{N}=\operatorname{diag}\left\{1 / \sigma_{x}^{2}, 1 / \sigma_{y}^{2}, 1 / \sigma_{\phi}^{2}\right\}, \boldsymbol{M}=\boldsymbol{I}$, where $\boldsymbol{I}$ denotes the identity matrix. The generated optimal trajectory is required to have a value of $J$ that is smaller than the straight line trajectory and for this reason it is advisable to choose the elements of the normalizing matrix $\boldsymbol{N}$ equal to the standard deviations of $\boldsymbol{P}$ on the straight line (at the final time step), namely $\sigma_{x}^{2}=0.15^{2} \mathrm{~m}^{2}, \sigma_{y}^{2}=0.15^{2} \mathrm{~m}^{2}, \sigma_{\phi}^{2}=2.16^{2} \mathrm{deg}^{2}$, computed by setting $N=0$. The WMR should finish with an error for the orientation angle less than $\phi_{\text {error }}=1 \mathrm{deg}$. The bigger $N$ is, the smaller the value of $J$ is. Table 1 contains the values of $\mathcal{U}, \mathcal{C}$, and $J$ (computed with $a_{1}=1, a_{2}=0.1$ ). As is obvious from 


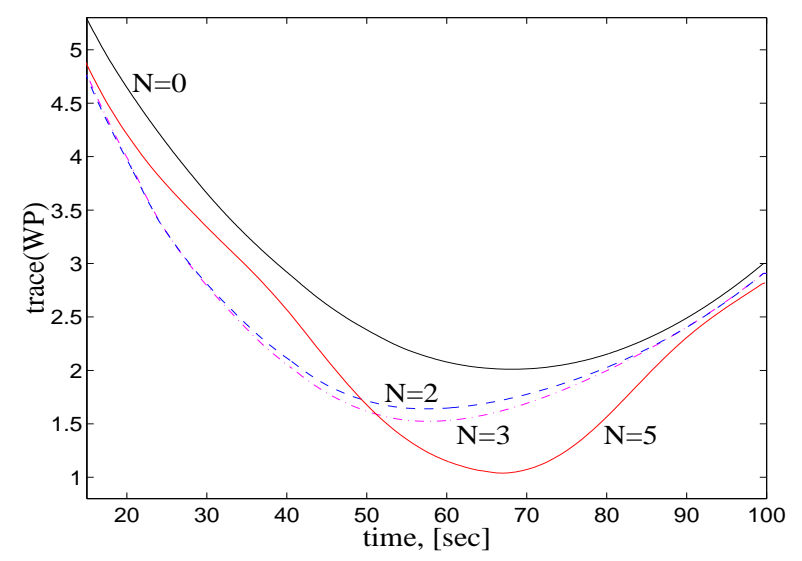

Figure 4: Evolution of $\operatorname{trace}(\boldsymbol{W P})$ in time

Table 1: Results with one beacon, $\mathcal{U}$ in the form (9)

\begin{tabular}{|c||c|c|c|}
\hline$N$ & $\mathcal{U}$ & $\mathcal{C}$ & $J$ \\
\hline \hline 0 & 3.00 & 1.00 & 3.10 \\
\hline 2 & 2.92 & 1.09 & 3.03 \\
\hline \hline 3 & 2.90 & 1.10 & 3.01 \\
\hline \hline 5 & 2.81 & 1.23 & 2.93 \\
\hline
\end{tabular}

Fig. 3, Fig. 4 and Table 1 the most accurate results at the goal configuration for $\mathcal{U}$ and $J$ are obtained with $N=5$ sinusoids. Better accuracy is provided with bigger $N$, at the cost of increased computational load. Nevertheless, even $N=3$ terms results in an improved final accuracy. The constrained optimization is performed using the "FMINCON" function of the Optimization Toolbox of MATLAB [14]. This function makes use of a sequential quadratic programming method.

Other amplitudes and trajectories have been generated using the criterion (11), by averaging within the interval $[30 \mathrm{sec}, 100 \mathrm{sec}]$. The normalization is performed with respect to the average standard deviations squared of the straight line. The trajectories are shown in Fig. 5, the average information criterion (11) is given in Table 2. For comparison, criterion (9) is also computed (with the obtained optimal amplitudes) and given in Fig. 6. Evidently, the general performance is considerably improved compared to the reference trajectory by using (11).

Results with two beacons are given in Fig. 7 and in Table 3. All initial conditions are the same as in the one beacon experiment, including the beacon position. Additionally another beacon is added. The plotted ellipses are $3 \sigma$ uncertainty ellipses. The standard deviations squared (at the end point on the straight line)

Table 2: Results with one beacon, $\mathcal{U}$ of the form (11)

\begin{tabular}{|c||c|c|c|}
\hline$N$ & $\mathcal{U}$ & $\mathcal{C}$ & $J$ \\
\hline \hline 0 & 3.00 & 1.00 & 3.10 \\
\hline 2 & 2.54 & 1.11 & 2.65 \\
\hline \hline 3 & 2.41 & 1.15 & 2.53 \\
\hline \hline 5 & 2.26 & 1.20 & 2.38 \\
\hline
\end{tabular}




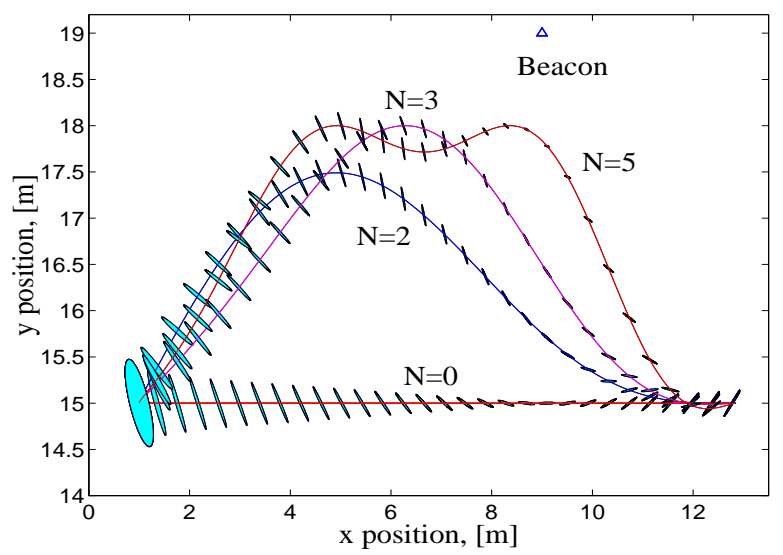

Figure 5: Trajectories, generated with (11) and (10)

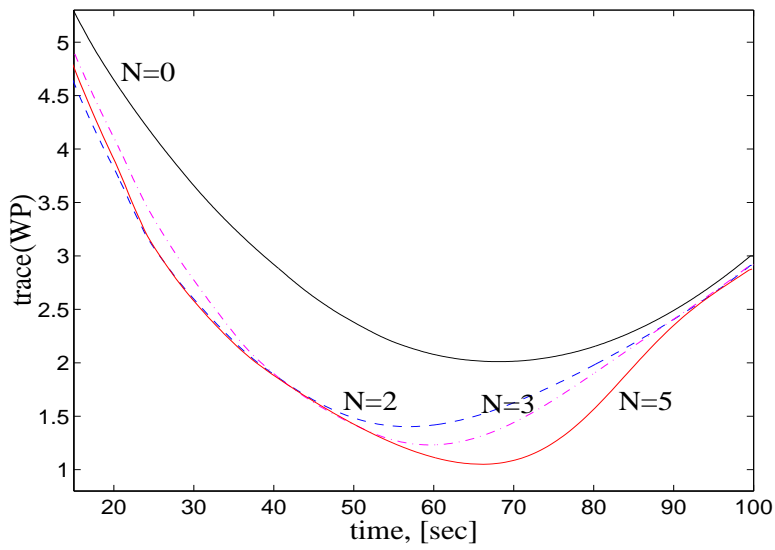

Figure 6: Evolution of $\mathcal{U},(9)$, for $N=0,2,3,5$

are: $\sigma_{x}^{2}=0.012^{2} m^{2}, \sigma_{y}^{2}=0.012^{2} m^{2}, \sigma_{\phi}^{2}=0.30^{2} \mathrm{deg}^{2}$. Obviously, more beacons placed in an appropriate way to cover well the whole area lead to considerable accuracy improvement. On the other side the computational load is augmented proportionally to the number of beacons.

Example 2. This example illustrates the approach application for a more complex trajectory. It is a pointto-point optimization. The initial and goal configurations are respectively $\boldsymbol{x}_{s}=(1 \mathrm{~m}, 15 \mathrm{~m},-155 \mathrm{deg})^{T}$, $\boldsymbol{x}_{g}=(8 \mathrm{~m}, 18 \mathrm{~m},-50 \mathrm{deg})^{T}$, keeping the same initial conditions and constants as in Example 1. Because the starting and goal orientation angle differ considerably, the WMR trajectory is generated as composed of two arcs and a straight line between them. There are few groups of possible robot trajectories, composed of straight line segments and tangential circular lines [19]. Two circles are passing through the starting point with a radius $R=L / \sin \left(\psi_{\max }\right)$. At the beginning the WMR performs a turn with a maximum steering angle, after that it moves on the trajectory, around the straight line connecting the two tangential points, and finally it turns again on a circular arc. So, this more complex trajectory is generated similarly to the straight line case, incorporating two extra circular segments. In general, to find the best path for each group of trajectories an optimization is performed and between the optimized trajectories (possible combinations of arcs and straight lines) could be chosen the one with the smallest criterion value. Fig. 8 shows one such generated trajectory, with criterion $\mathcal{U}$ in the form (9). A trajectory generated in the presence of a known 


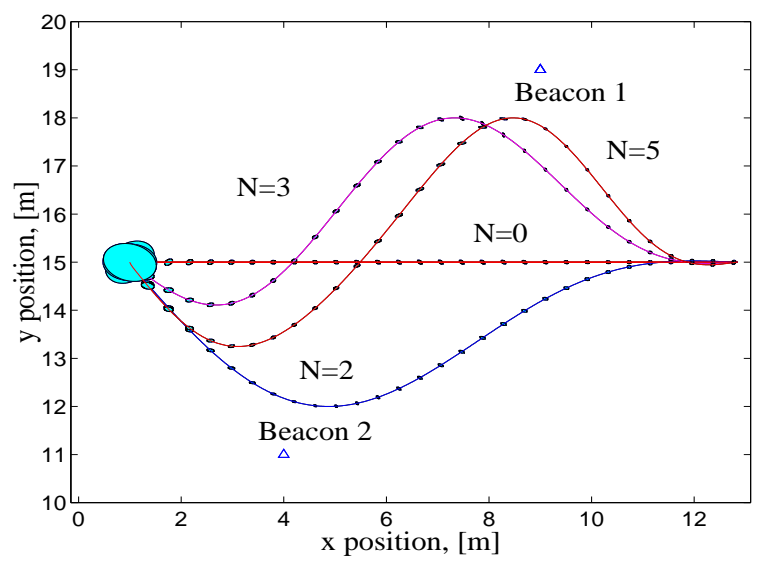

Figure 7: Trajectories, generated with (9) and (10)

Table 3: Results with two beacons, $\mathcal{U}$ of the form (9)

\begin{tabular}{|c||c|c|c|}
\hline$N$ & $\mathcal{U}$ & $\mathcal{C}$ & $J$ \\
\hline 0 & 3.00 & 1.00 & 3.10 \\
\hline 2 & 2.74 & 1.17 & 2.86 \\
\hline 3 & 2.72 & 1.24 & 2.84 \\
\hline 5 & 2.59 & 1.34 & 2.72 \\
\hline
\end{tabular}

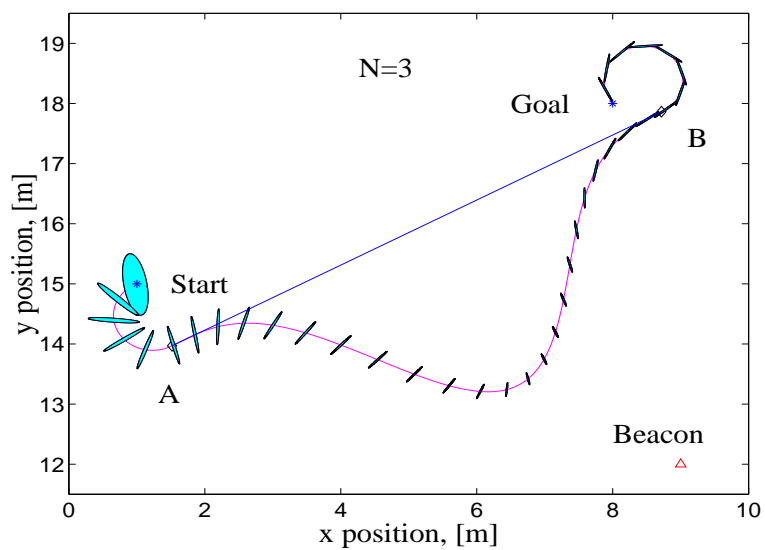

Figure 8: WMR trajectory

obstacle, with allowed threshold distance between the WMR and the obstacle's surface $d_{o, \min }=0.45 \mathrm{~m}$ is plotted in Fig. 9. As it can be seen from Fig. 9, the constraint (16) for the distance to the obstacle is respected.

The computation of the control parameters (steering angle and linear velocity) for the different types of trajectories is given in the Appendix. 


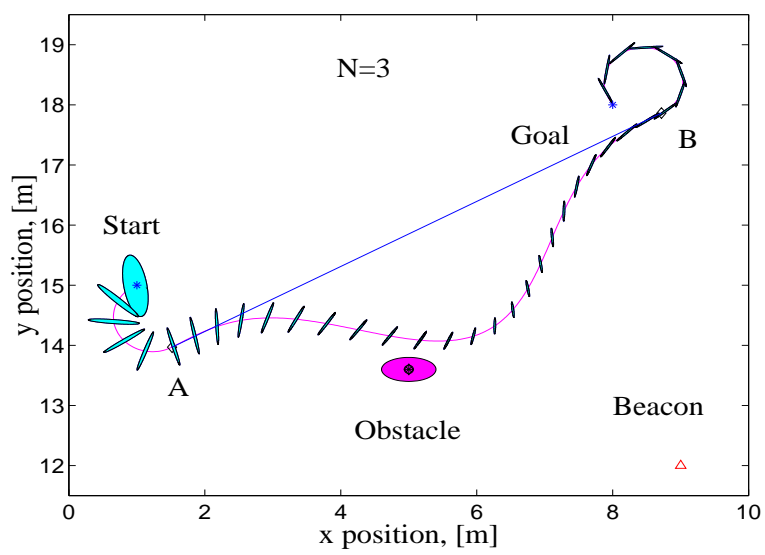

Figure 9: Trajectory in the presence of an obstacle

\section{Discussion}

Throughout the paper a multisine approach for trajectory optimization has been developed and applied to a nonholonomic WMR. As it has been shown the generated trajectories are smooth, always obeying the position constraints at the starting and goal configurations. The approach has been applied with landmarks (beacons) with known positions. Nevertheless, generalization for the case of unknown beacons' positions is straightforward. The beacons' positions then should be estimated together with the system states like in [20]. By active sensing the robot is approaching to the beacons (obvious from the results in the examples), that is a distinction from a movement over a reference trajectory.

A performance criterion has been defined and applied to trajectory optimization. The criterion for active sensing has the following features:

- it incorporates information and cost parts;

- the influence of the different factors is decoupled;

- the information term characterizes information extraction and accuracy at the goal configuration, which the WMR is required to reach;

- it is invariant to physical units and is formulated in an appropriate way to gain information from the measurements;

- the cost term represents the ratio between the final time for reaching the goal configuration and the respective time for moving over the modified trajectory;

- observing the criterion, conclusions can be drawn about the accuracy, as well as the convergence rate of the optimization procedure;

- it provides an optimization over the whole trajectory (global optimization procedure);

- the criterion is minimized, while satisfying nonholonomic, physical and boundary constraints;

- the presence of known obstacles is accounted by adding additional constraints to the optimization;

- the simulation results obtained even with small number of sine functions, $N=3$, show very good accuracy at the goal configuration;

- the approach has been applied to a nonholonomic WMR, but it is general and can be implemented to other systems. It is suitable both for simple trajectories (line segments or arcs), and more complex ones. Its effectiveness has been demonstrated through simulation studies. A next step for future work is to implement it in a real experimental set up. 


\section{Conclusions}

This paper introduces an information-based approach for trajectory optimization of a robot moving between two configurations. The solution is searched within a preset class of functions, namely those of the harmonics. The optimal trajectory represents a linear combination of sine functions. The problem has been examined as a global optimization subject to constraints. Both cases with and without reference trajectory, with one and more sensors have been considered. It is shown that more sensors located in an appropriate place improve considerably the accuracy. The effectiveness of the approach is illustrated by different simulation results with a WMR in environment with and without obstacles.

Acknowledgments. Herman Bruyninckx is a Postdoctoral Fellow of the F.W.O-Vlaanderen, Belgium. Financial support by K. U. Leuven's Concerted Research Action GOA/99/04 and the Center of Excellence BIS21 grant ICA1-2000-70016 are also acknowledged.

\section{A Appendix. Computation of the control parameters for an arbitrary reference trajectory}

Given the reference trajectory, $\left(x_{r, k}, y_{r, k}, \phi_{r, k}\right)^{T}$, the angle $\beta_{k}$ between its tangent and the $x$ axis is (Fig. 1)

$$
\beta_{k}=\arctan \frac{\Delta y_{r, k}}{\Delta x_{r, k}}
$$

and the angle $\alpha_{k}$ between the reference and modified trajectory then is

$$
\alpha_{k}=\arctan \frac{\Delta y_{k}}{\Delta x_{k}}-\beta_{k}
$$

The modified trajectory is provided by equations

$$
\begin{aligned}
& x_{k}=x_{r, k}+l_{k} \cos \left(\beta_{k}+\frac{\pi}{2}\right), \\
& y_{k}=y_{r, k}+l_{k} \sin \left(\beta_{k}+\frac{\pi}{2}\right) .
\end{aligned}
$$

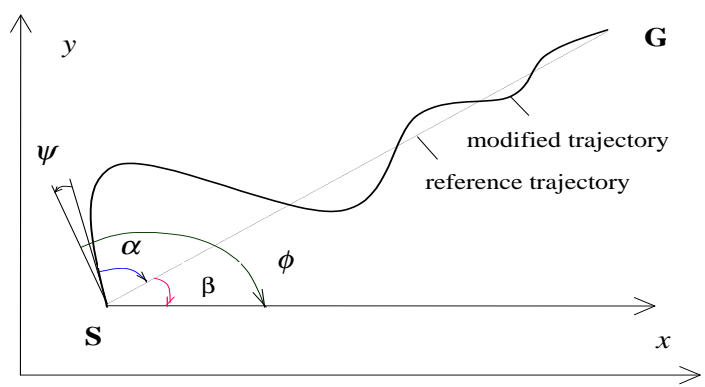

Figure 10: WMR trajectory

The steering angle is obtained from

$$
\psi_{k}=\phi_{k}-\alpha_{k}-\beta_{k},
$$

and the linear velocity from

$$
v_{k}=\frac{1}{T} \sqrt{\Delta s_{r, k}^{2}+\Delta l_{k}^{2}}=\sqrt{v_{r, k}^{2}+\frac{\Delta l_{k}^{2}}{T^{2}}}
$$


where $\Delta s_{r, k}=s_{r, k}-s_{r, k-1}, \Delta l_{k}=l_{k}-l_{k-1}$.

In particular, for a straight line reference trajectory, not parallel to $x$ axis (Fig. 10), we get $\beta_{k}=$ const, $x_{r} \neq$ const, $\psi_{r, k}=0$. For for a straight line reference trajectory, parallel to $x$ axis, $\beta_{k}=0, x_{r}=$ const , $\psi_{r, k}=0$.

\section{References}

[1] Y. Bar-Shalom and X.R. Li, Estimation and tracking: Principles, techniques and software, Artech House, 1993.

[2] O. Brock and O. Khatib, Elastic strips: Real-time path modification for mobile manipulation, Proc. of the Eighth Int. Symp. of Robotics Research, 1997.

[3] __ Elastic strips: A framework for integrated planning and execution, Proc. of 1999 Int. Symp. of Exper. Rob., 1999, pp. 245-254.

[4] _ High-speed navigation using dynamic window approach, Proc. of Int. Conf. on Rob. and Automation, 1999, pp. 245-254.

[5] W. Burgard, D. Fox, and S. Thrun, Active mobile robot localization by entropy minimization, Proc. of 2nd Euromicro Worksh. on Adv. Mobile Rob., 1997.

[6] G. Calafiore, M. Indri, and B. Bona, Robot dynamic calibration: Optimal trajectories and experimental parameter estimation, IEEE Trans. on AC 13 (1997), no. 5, 730-740.

[7] J. De Geeter, J. De Schutter, H. Bruyninckx, H. V. Brussel, and M. Decreton, Tolerance-weighted L-optimal experiment design: a new approach to task-directed sensing, Adv. Rob. 13 (1999), no. 4, 401-416.

[8] D. Fox and W. Burgard, Active Markov localization for mobile robots, Robotics and Autonomous Systems 25 (1998), no. 4, 195-207.

[9] S. Julier, The scaled Unscented transform, Proc. of Amer. Contr. Conf., 2002, pp. 4555-4559.

[10] S. Julier, J. Uhlman, and H. Durrant-Whyte, A new method for the transformation of means and covariances in filters and estimators, IEEE Trans. on AC 45 (2000), no. 3, 477-482.

[11] R. Kalman, A new approach to linear filtering and prediction problems, Trans. ASME, J. Basic Eng. (1960), 35-45.

[12] J.-P. Laumond, Robot motion planning and control, Springer-Verlag, 1998.

[13] J.-P. Laumond, P. Jacobs, M. Tä̈x, and R. Murray, A motion planner for nonholonomic mobile robots, IEEE Trans. on Robotics and Automation 10 (1994), no. 5, 577-592.

[14] The MathWorks, The optimization toolbox user's guide, MA, 2001.

[15] L. Mihaylova, H. Bruyninckx, and J. De Schutter, Active sensing of a wheeled mobile nonholonomic robot, Proc. of IEEE Benelux Signal Processing Symposium (Leuven, Belgium), 21-22 March, 2002, pp. $125-128$.

[16] L. Mihaylova, J. De Schutter, and H. Bruyninckx, A multisine approach for trajectory optimization based on information gain, Proc. of the IEEE/RSJ International Conf. on Intelligent Robots and System (Lauzanne, Switzerland), Sept. 30 - Oct. 4, 2002.

[17] A. Mutambara, Decentralized estimation and control for multisensor systems, CRC Press, 1998.

[18] S. Quinlan and O. Khatib, Elastic bands: Connecting path planning and control, Proc. of IEEE Conf. on Rob. and Autom., Vol. 2, 1993, pp. 802-807. 
[19] J. Reeds and L. Shepp, Optimal paths for a car that goes both forwards and backwards, Pacific Journ. of Mathemat. 145 (1990), no. 2, 367-393.

[20] M. Ribo, S. Brahim-Belhouari, and L. Pronzato, Motion planning for the precise location of a mobile robot, Proc. of Europ. Contr. Conf., 1997.

[21] N. Roy, W. Burgard, D. Fox, and S. Thrun, Coastal navigation - mobile robot navigation with uncertainty in dynamic environments, Proc. of the IEEE Int. Conf. on Rob. and Autom., 1999.

[22] S. Sekhavat and J.-P. Laumond, Topological property for collision-free nonholonomic motion planning: the case of sinusoidal inputs for chained form systems, IEEE Trans. on Rob. and Automation 14 (1998), no. $5,671-680$.

[23] C.E. Shannon, A mathematical theory of communication, I and II, The Bell System Technical Journal 27 (1948), 379-423 and 623-656.

[24] J. Swevers, C. Ganseman, D. Tukel, J. De Schutter, and H. V. Brussel, Optimal robot excitation and identification, IEEE Trans. on AC 13 (1997), no. 5, 730-740.

[25] S. Thrun, Monte Carlo POMDPs, Advances in Neural Inform. Proc. Syst. 12, 1999, pp. 1064-1070.

[26] E. Wan and R. van der Merwe, The Unscented Kalman filter, Kalman Filtering and Neural Networks (S. Haykin, ed.), Wiley Publ., 2001. 


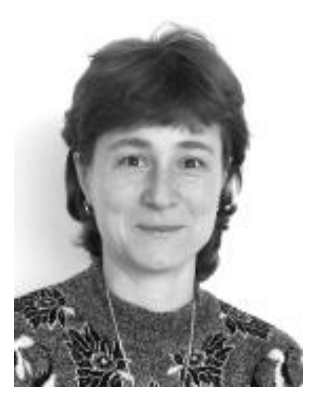

Lyudmila Mihaylova obtained a M.S. and Ph.D. degrees in Systems and Control Engineering from the Technical University of Sofia, Bulgaria in 1989 and 1996, respectively. Since 1996-2000 she has been working at the Bulgarian Academy of Sciences as a Research Scientist and at the same time as a part-time Assistant Professor at Technical University of Sofia. Since September 2000 she joined Katholieke Universiteit Leuven, Belgium, Department of Mechanical Engineering, where currently is a Research Associate. Now she is involved in the development and application of advanced techniques for filtering and estimation of nonlinear stochastic systems under uncertainties, machine learning, active sensing. Other her areas of interest are system identification and control, sensor data fusion. Dr. L. Mihaylova is a member of the IEEE, the Signal Processing Society.

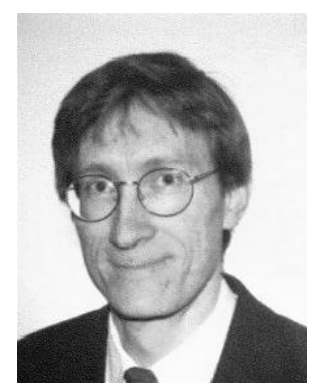

Joris De Schutter received the degree of mechanical engineer from the Katholieke Universiteit Leuven, Belgium, in 1980, the M.S. degree from the Massachusetts Institute of Technology, in 1981, and the Ph.D. degree in 1986 in mechanical engineering, from Katholieke Universiteit Leuven. Following work as a control systems engineer in industry, in 1986, he became a Lecturer in the Department of Mechanical Engineering, Division PMA (Production, Machine Design and Automation), Katholieke Universiteit Leuven where he is a full Professor since 1995. He teaches courses in kinematics and dynamics of machinery, control and robotics. His other area of interests are force control, compliant motion, position control of flexible systems and drive systems, data fusion. Prof. De Schutter is a member of the IEEE.

Herman Bruyninckx obtained the Bachelor's degree in Mathematics (1984), M.Sc. degree in Computer Science (1987) and in Mechatronics (1988), all from Katholieke Universiteit Leuven, Belgium. In 1995 he got his $\mathrm{PhD}$ dergee in Applied Sciences from the same university. His thesis is devoted to force controlled compliant robot motion. Since 1994 he is a Post-Doctoral Researcher with the Fund for Scientific ResearchFlanders (F.W.O) in Belgium, and since 1995 he is an Assistant Professor at the Department of Mechanical Engineering (KUL). His research interests include on-line estimation of model uncertainties in sensor-based 


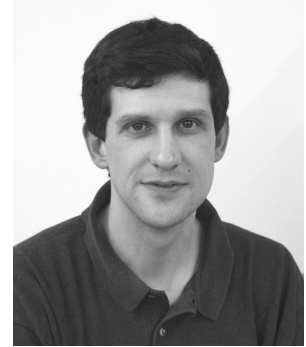

robot task, kinematics of robots, geometric foundations of robotics compliant robot motion, active sensing, motion planning. Dr. Bruyninckx is a member of the IEEE. 